\title{
The Optimization of Genetic Algorithm in Wireless Sensor Network Coverage
}

\author{
Wengen Gao, Qigong Chen ", Ming Jiang, Yunfei Li and Shinong Wang \\ AnHui Key Laboratory of Detection Technology and Energy Saving Devices, \\ AnHui \\ Polytechnic University, 241000, WuHu, AnHui, China \\ ahpuchina@ahpu.edu.cn,Prof_ChenQigong@163.com, \\ Prof_Jiangming@163.com, \\ nathonlee@163.com,wsn_1111@163.com
}

\begin{abstract}
According to the maximum coverage problem in wireless sensor networks, GA algorithm combined with the standard processing method can improve the network coverage based on, but there is a risk of falling into local optimum, and costs more computation time. On the condition of analysis and proof of the effectiveness by the employment of normalization processing to resolve coding redundancy of MCSDP, further details of the evaluate conditions are proposed, and new parent selection mechanism is introduced, which both are verified by compared experiments. The experiment results show that the optimization processing method proposed in this paper retains the characteristics of existing methods, and has better optimization performance and improves the network coverage rate as well as calculation speed, which verifies the effectiveness and superiority of the method proposed in this paper.
\end{abstract}

Keywords: Genetic algorithm; Maximum coverage; New parent selection mechanism; Solution space

\section{Introduction}

The wireless sensor network is widely employed in localization and trace, etc. and provides human the accurate environment information. In the detection system of air quality, earthquake prediction system, the wireless sensor technology takes an important part to prepare people better for the natural disaster and protect own lives and property. It is still necessary to consider that how to sense the largest scope with the least sensors, though the price of sensors declines with the improvement of the technology.

Some research consider on enlarging the sensing coverage in static sensors [1-4], and these research focus on the mobile phone field. Some research emphasizes the problem hoe to utilize the cheap static sensors and the relatively expensive mobile sensors to establish the low cost and large coverage sensor network [5], and some study on how to monitor the goals effectively [6]. The optimal employment localization of the sensors benefits us with more sufficient information of the monitor goal in the condition of reducing the manpower [7-9]. In this research field, it is considered that how to get efficiently the maximum sensing coverage with the given sensors type and amount [10-12]. 


\section{Problem Description}

\subsection{Assumption}

The coverage model of the geometrical relationship between the sensor and the sensing point is chosen to measure the sensor quality and ability. The common coverage model is:

$$
f(d(s, x))=\left\{\begin{array}{cl}
1 & d((s, x)) \leq r_{s} \\
0 & \text { else }
\end{array}\right.
$$

The distance between the sensor $s$ and the sensing point $x$ is presented as $d(s, x)$, the constant $r_{s}$ indicates the sensing range of the sensors. This model is omnidirectional, and the sensing area is discoid. If the sensing point is in the sensing range, the model value is 1 , otherwise is 0 . Different sensor type has different sensing range.

According to the contents of research, the coverage in the sensor network can be divided into three categories: the regional coverage, the impedimental coverage and the objective coverage. The sensors are regarded as the same, and the Boolean model is employed to process the regional coverage problem. It is considered that how to maximum the coverage range with the certain sensor amount. A certain coverage problem is assumed, and the coverage method proposed in the paper, is easily applied in other coverage problem with change of the evolution-function.

\subsection{Problem Description}

There are $k$ kinds of static sensors, and every kind of sensors has been set, and the sensing radiuses are $r_{1}, r_{2}, \ldots, r_{k}$. There are $n$ sensors of all kinds in amount. It is assumed that there is one sensor at least of every kind, and the numbers of sensors $i$ type are from $n_{i}$ to $n_{i+1}-1$. The given $k+1$ sensors meet $n_{k+1}=n+1$ and $n(=1)<n_{2}<\ldots<n_{k+1}(=n+1)$, and the kind of $i$ has $n_{i+1}-n_{i}$ sensors, and $i=1,2, \ldots k$.

The location $\left(x_{1}, y_{1}\right),\left(x_{2}, y_{2}\right), \ldots\left(x_{n}, y_{n}\right)$ will be found to get the maximum coverage area with $n$ sensors meet the field $A$.

The circle $C_{r}(x, y)$, locating its center at $(x, y)$, has the radius $r$, and it is expressed as:

$$
C_{r}(x, y):=\left\{(v, w) \in R^{2}:(v-x)^{2}+(w-y)^{2} \leq r^{2}\right\}
$$

and can also be described as:

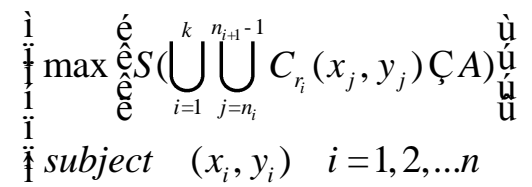

$r_{i}$ is positive real number, $1 \leq i \leq k$, is incremental positive number, $1 \leq i \leq k+1, n_{1}=1, n_{k+1}=n+1, A$ is two-dimension field [13].

\subsection{Solution Domain}

In this example, the coverage area does not change while the sensor's parameters change of the same type. The same solving progress can be carried out in more than one coding scheme. Hence, the coding scheme and the solution domain are different. 
Make $\mathrm{G}$ be the coding set, and $\mathrm{g}\left(g_{1}, g_{2}, \ldots g_{n}\right), \mathrm{h}\left(h_{1}, h_{2}, \ldots h_{n}\right)$, and are Euclidean distance. The equivalence symbol is defined as $\sim$ :

Definition 1: $x \sim y$ if and only if the sequence $\sigma_{i} \in \sum_{n_{i+1}-n_{i}}$, and $i \leq k . \sigma_{i} \in \sum_{n_{i+1}-n_{i}}$ is a sequence with the length of $n_{i+1}-n_{i}$, and $\sigma(x)$ is $\sigma$ sequence of $x$.

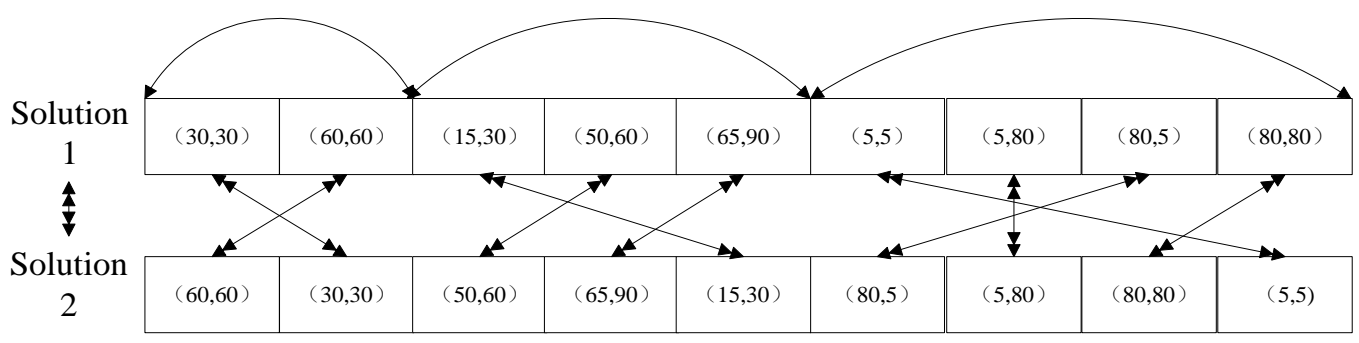

Figure1. The Comparison of the Two Equivalent Solutions

$n_{i+1}-n_{i}$ is the amount of the type $i$ sensor. $\left(g_{n_{i}}, g_{n_{i+1}}, \ldots g_{n_{i+1}-1}\right)$ is the coordinate sequence of type $i$ sensor. The solution 1 and solution 2 is equivalent as shown in Fig.1. $\left(n_{2}-n_{1}\right) !\left(n_{3}-n_{2}\right) ! \ldots .\left(n_{k+1}-n_{k}\right)$ ! is the equivalent expression of $g \in G$. According to the definition $1, G / \sim$ is the solution set of coded set.

The coded set $\mathrm{G}$ is established by employing the intelligent algorithms e.g. genetic algorithm, neural network, as well as its equivalent solution $G / \sim$. So the efficient method is required to find the solution $G / \sim$ in the coded set.

\section{The Optimization of Normalization}

\subsection{Genetic Structure}

The genetic structure is described based on the contents of the paper. The population is $\mathrm{N}$, and randomly sampled N/2 sets. Each set produces N/2 offspring through crossing and mutating. The best $\mathrm{N}$ samples are chosen to be the genetic male parent of the next generation by disorganizing the male parent and children. In the experiment, the population is 50 , namely $\mathrm{N}=50$ and the genetic mutation is set at 1000 generations. The cross parameter and mutation parameter are respectively $B L X-\alpha$ and Gaussian.

\subsection{Evaluation Function}

The purpose of the evaluation function is to solve the coverage range of the sensor. Therefore, the results are calculated by resampling in Monte Carlo. $X$ is the coverage area, and:

$$
\begin{gathered}
\bigcup_{i=1}^{k} \bigcup_{j=n_{i}}^{n_{i+1}-1} C_{r_{i}}\left(x_{j}, y_{j}\right) \cap A \\
S(X)=\iiint_{A} I_{X}(x, y) d x d y=\lim _{L \rightarrow \infty} \frac{S(A)}{L} \sum_{l=1}^{L} I_{X}\left(\tilde{x}_{l}, \tilde{y}_{l}\right)
\end{gathered}
$$

In (5), $I_{X}(\cdot)$ is defined as:

$$
I_{X}(x, y)= \begin{cases}1 & (x, y) \in X \\ 0 & (x, y) \notin X\end{cases}
$$


$\left(\tilde{x}_{l}, \tilde{y}_{l}\right)$ is the point of the field A. It can assess whether the sample $\left(\tilde{x}_{l}, \tilde{y}_{l}\right)$ is in the range of the sensor by solving the value of $I_{X}\left(\tilde{x}_{l}, \tilde{y}_{l}\right)$ corresponding to each $l=1,2, \ldots L$. Therefore, the maximum time complexity of Monte Carlo evaluation function is $O(n L)$. With more samples, the estimation results are more accurate. As shown in the Fig.2.

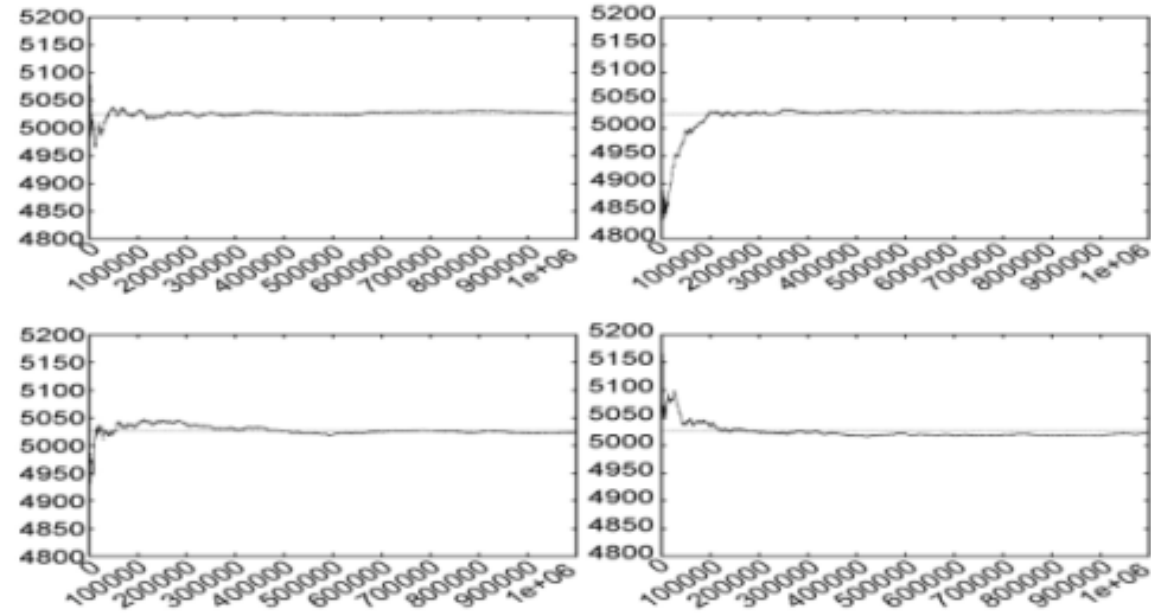

Figure 2. The Relationship between Samples and Estimation Value

However, calculated quantity of GA is more with more samples, namely the operating cost of estimation results will be higher. Hence, it is more effective to reduce the operating cost than improve the accuracy of the estimation results with the large quantity of samples. Based on the analysis, the employed calculation method is calculating a small number of samples at the initial stage, and the calculating samples increase with the augment of the genetic algebra.

\subsection{Normalization}

The coded set and solution set are different because of the coding redundancy. The solution set $P$ is related to the entropy $G / \sim$, and $G$ is the coded set domain. The normalization is utilized to lower the impact of the redundancy on the calculation. The normalization transfers the coded set of the male parent into another coded set.

Assumption: make $g=\left(g_{1}, g_{2}, \ldots, g_{n}\right)$ and $h=\left(h_{1}, h_{2}, \ldots, h_{n}\right)$ subject G. $g_{i}$ and $h_{i}$ are 2-D collaboration, and $i=1,2, \ldots n$. Make

$$
D(g, h)=\sum_{i=1}^{n} d\left(g_{i}, h_{i}\right)
$$

It is one matrix of $\mathrm{G}$, and $\mathrm{d}$ is one matrix of $R^{2}$. So,

$$
\tilde{D}(g, h):=\min _{\sigma \in \prod_{i=1}^{k} S_{n_{i+1}-n_{i}}} D(g, \sigma(h))
$$

It is one matrix of $G / \sim$.

Demonstration: $s$ is made be one value of the set $\prod_{i=1}^{k} S_{n_{i+1}-n_{i}}$. The number of the addend has no relation with the result for the addend is limited when $D(g, h)=\sum_{i=1}^{n} d\left(g_{i}, h_{i}\right)$ is calculated. Therefore, $D(g, h)=D(\sigma(g), \sigma(h))$. So each $s$ in the coded set is equidistant as well as $\prod_{i=1}^{k} S_{n_{i+1}-n_{i}}$. The relational symbol $\sim$ represents the equivalence relation. Hence, $\tilde{D}(g, h)$ is a matrix of $G / \sim$. 
Therefore, if the normalization is not adopted to solve the MCSDP with the application of GA, the GA will only find the coded set, and will not affect the original solution. Changing the parameters of the same kind of sensors does not influence the solution set in MCSDP. So the appropriate rearrangement of the gene does not change the original solution, and makes the search to the solution domain more efficient. A normalization way to the MCSDP is proposed.

Literature [13] made comparisons of the effects between maximization, random and minimization, and research results show that minimization process has the best effect in terms of employing GA algorithm to solve MCSDP. Therefore, on the basis of literature [13], to further normalization method optimization was proposed in the paper. First, the algorithm randomly generated initial parents, and uses the appropriate method to reorder crossover and mutation, and chooses from the N/2 samples with good effect to be the next parents. The normalization flow diagram of the optimized processing step is shown in figure 3 .

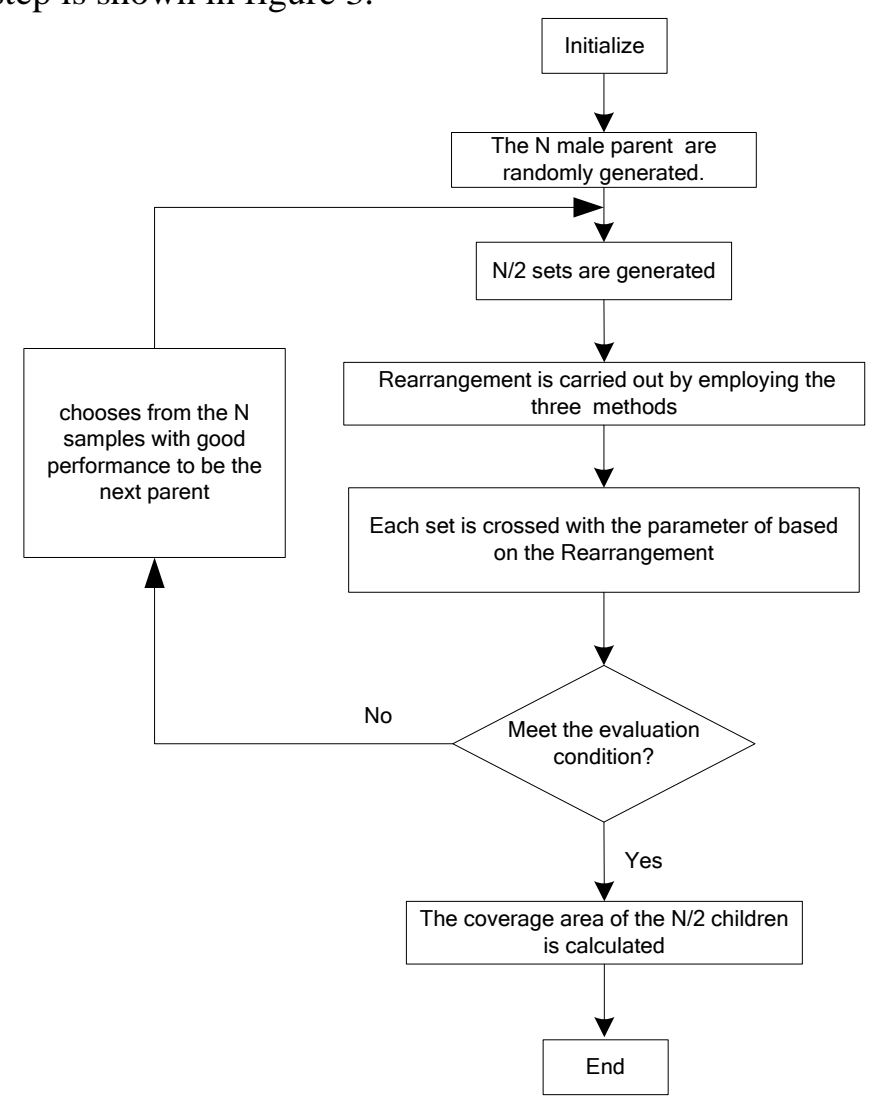

Figure 3. The Flow Chart of Normalization Processing Steps

\section{Experiment Results}

In order to verify the effectiveness and superiority of the proposed optimization method, experimental environment is consistent with the [13], and the experiment results compared with the experimental results in [13]. The experiment environment is as:

It is assumed that $A=[0,100] \times[0,100]$, and three kinds of sensors are employed, and the radius of every kind is respectively $r_{1}, r_{2}$ and $r_{3}$, meanwhile, the radiuses meet the demand $r_{1}=0.8 \times r_{2}, r_{2}=0.8 \times r_{3}$. The amount of every kind sensor is related to the coverage compactness $\alpha, \alpha=0.5$. 
The Exp. 1 is under the method proposed in this paper, and the Exp.2 adopts the method of [13]. The experiment results are shown in Fig.4 to Fig.9, and the corresponding relationship between the sensor and the x-axis is shown in Table.1.

Table.1 The corresponding relationship between the sensor and the x-axis

\begin{tabular}{|c|c|c|c|c|c|c|c|c|c|c|c|c|}
\hline No. & 1 & 2 & 3 & 4 & 5 & 6 & 7 & 8 & 9 & 10 & 11 & 12 \\
\hline Instance & S1-0.7 & S2-0.7 & S3-0.7 & S4-0.7 & S1-0.8 & S2-0.8 & S3-0.8 & S4-0.8 & S1-0.9 & S2-0.9 & S3-0.9 & S4-0.9 \\
\hline
\end{tabular}

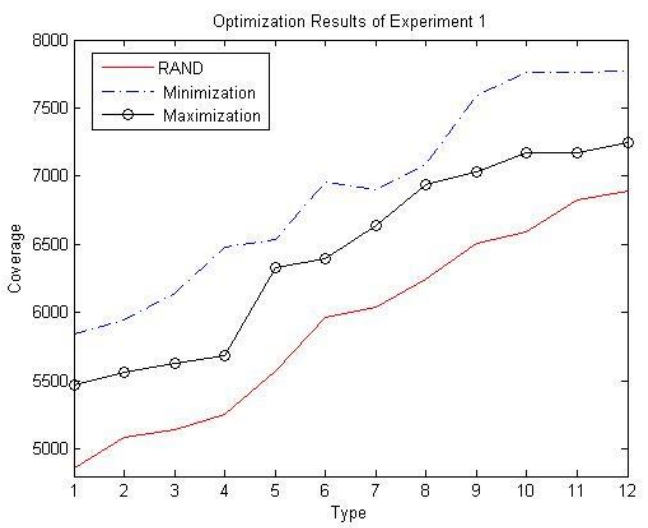

Figure 4. The Comparision of Experiment Results between Three Methods in Exp.1

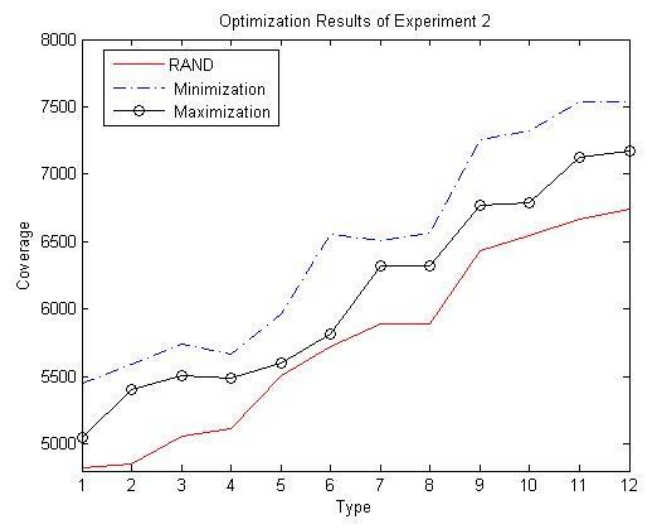

Fig.5 The Comparison of Experiment Results between Three Methods in Exp.2

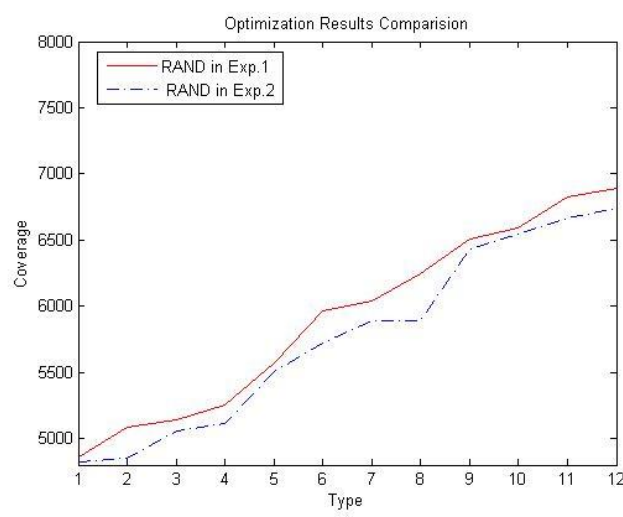

Figure 6. Result Comparison of Randomnes Processing Method between Exp.1 and Exp.2 


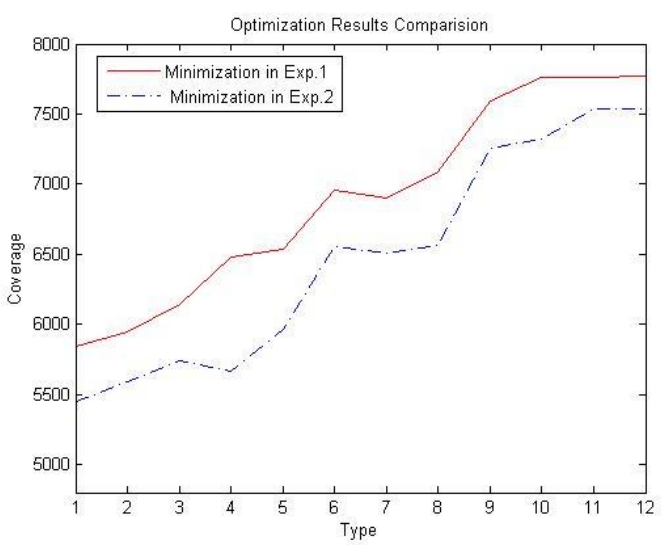

Figure 7. Result Comparison of Minimization Processing Method between Exp.1 and Exp.2

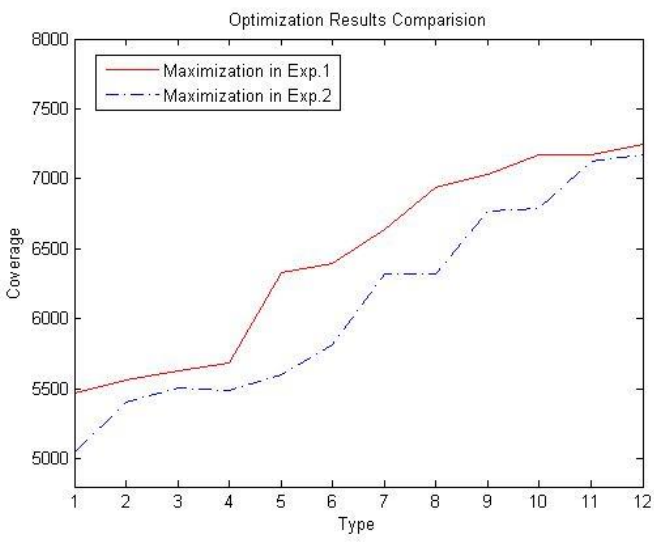

Figure 8. Result Comparison of Maximization Processing Method between Exp.1 and Exp.2

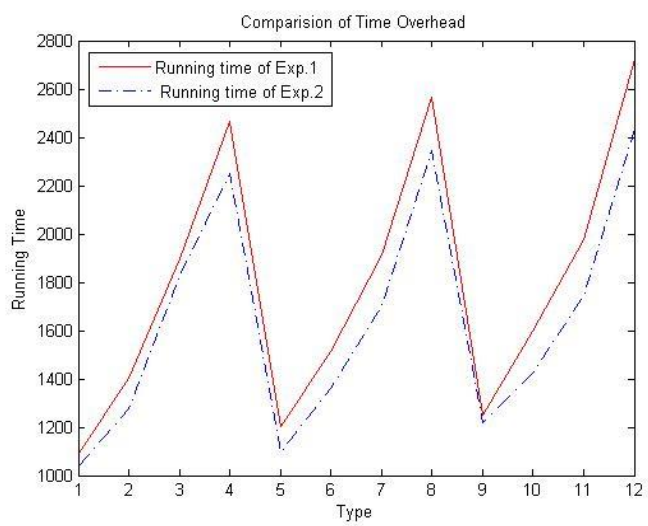

Figure 9. The Comparison of Time Overhead of Minimization Method between Exp.1 and Exp.2

In Fig.4 and Fig.5, there are the optimization method proposed in this paper and the method proposed in [13], and the characteristics of the two methods, which shows that minimization has the best performance, and the random processing is the worst, are consistent with each other. The random processing method randomly selects samples, and cannot inherit the characteristics from the parent generation; however, the minimum processing method retains the father generation characteristics at the extreme, which leads to the best performance. 
Based on Fig.6, Fig.7 and Fig.8, with regard to the three kinds of random, minimization, maximization methods, the optimization method proposed in this paper has better performance than the proposed method in [13]. On the basis of method proposed in [13], further details of the evaluate conditions are proposed, and new parents selection mechanism is introduced. The samples with good performance are randomly selected to be the next parent, which not only reduces the parent sample range, but also ensure the excellent father characteristics. The new proposed method can effectively reduce the probability of trapping in local optimum, and can produce offspring with more coverage to improve the coverage area of the whole network. At the same time, the new proposed method has a faster optimization speed, and saves processing time overhead. The comparison of running time between two experiments of minimization method is shown in Fig.9.

\section{Conclusions}

This paper proved that the validity of introduction of normalization in processing of coding redundancy resolution of MCSDP based on GA algorithm, and optimization are based on the existing normalization method, further details of the evaluate conditions are proposed, and new parent selection mechanism is introduced, which both are verified by experiments. Experiment results show that the optimization processing method proposed in this paper retains the characteristics of the existing methods, and has better optimization performance and improves the network coverage rate and the speed of calculation, which validates the effectiveness and superiority of the method proposed in this paper.

\section{Acknowledgements}

The paper was supported by Natural Science Foundation of China (61172131; 61271377), and extend sincere gratitude to prof. Chen and research team.

\section{Reference}

[1] A. Howard, M. J. Matarić and G S. Sukhatme, "An incremental self-deployment algorithm for mobile sensor networks", Autonomous Robots, vol. 13, no. 2, (2002), pp. 113-126.

[2] I. Yamada, "The hybrid steepest descent method for the variational inequality problem over the intersection of fixed point sets of nonexpansive mappings", Studies in Computational Mathematics, vol. 8, (2001), pp. 473-504.

[3] Z. Shen, Y. Chang, H. Jiang, et al., "A generic framework for optimal mobile sensor redeployment [J]. Vehicular Technology", Transactions on Vehicular Technology, vol. 59, no. 8, (2010), pp. 4043-4057.

[4] Y. Zou and K. Chakrabarty, "Sensor deployment and target localization based on virtual forces", Twenty-Second Annual Joint Conference of the IEEE Computer and Communications, Societies, (2003).

[5] G. Wang, G. Cao and T. LaPorta, "A bidding protocol for deploying mobile sensors", Proceedings. 11th IEEE International Conference on Mobile Ad-Hoc and Sensor Systems, (2003).

[6] H.Li, M. Y. Chow and Z. Sun, "State feedback stabilization of networked control systems", IET Control Theory and Applications, (2009), vol.3, no. 7,pp. 929-940.

[7] D. S. Kim, Y. S. Lee, W. H. Kwon, et al., "Maximum allowable delay bounds of networked control systems", Control Engineering Practice, (2003), vol. 11, no. 11, pp. 1301- 1313.

[8] D. Yue, Q. L. Han, C. Peng, "State feedback controller Design of networked control systems", Transactionson Circuits and Systems II: Express Briefs, vol. 51, no. 11, (2004), pp. 640-644.

[9] X. F. Jiang, Qing Long, Shi Rong Liu, et.al,. "A new H-infinity stabilization criterion for networked control systems", Transactions on Automatic Control, (2008), vol. 53, no. 4, pp. 10251032.

[10] S. Soliman, M. Tan, Guanzheng, "Conditional sensor deployment using evolutionary algorithms", Journal of Convergence Information Technology, vol. 5, no. 2, (2010), p 146-154.

[11] M. Fengying, "Sensor deployment optimization based on simulation of gas distribution in underground fully mechanized coal face”, Advanced Materials Research, vol. 503-504, (2012), p $1468-1471$. 
[12] T. Clouqueur, V. Phipatanasuphorn, P. Ramanathan,; K. K Saluja, "Sensor deployment strategy for target detection", Proceedings of the ACM International Workshop on Wireless Sensor Networks and Applications, (2002), p 42-48.

[13] M. Mohamed Aadhil, J. Roselin, M. E., "Maximizing Coverage Problem Using Genetic Algorithm in Wireless Sensor Network", International Journal of Emerging Technology and Advanced Engineering, (2014), vol. 4, no. 2, pp. 2250-2459.

\section{Authors}

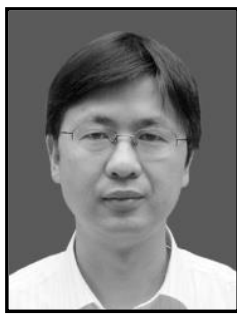

Wengen Gao, a doctor student in Jiangnan University, and is a senior lecturer of the School of Electrical Engineering. He has published quite large numbers of articles in internaltional conference, and his main research araes focus on WSN and algortithms.

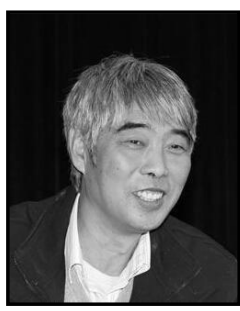

Qigong Chen, the chief director of AnHui Key Laboratory of Detection Technology and Energy Saving Devices, the academic leader of AnHui key subject 'Detection Technology and Automatic Devices', and the professor of doctoral supervisor, received his master degree in Zhejiang University in 1988, and enjoys the special allowance of AnHui Province. He is the senior member of Chinese Society of Electrical Engineering, and has been researching the electric automation, intelligent observation and control technology, and Networked Control System for long time.

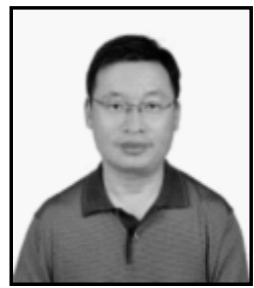

Ming Jiang, He was born in 1965 in Wuhu, Anhui, China, and received his master degree at Shanghai University. He is the vice director of AnHui Key Laboratory of Detection Technology and Energy Saving Devices, and a professor in Cyber Physical System.

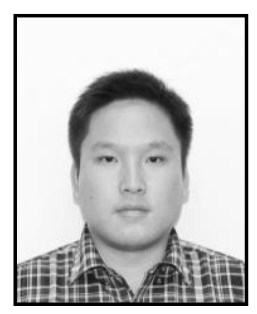

Yunfei Li, He was born in 1989 in Anqing, Anhui, China, and studys for his master degree at Anhui Polytechnic University. His research area is wireless sensor network localization.

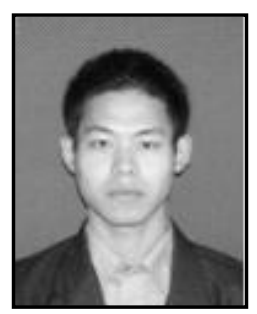

Shinong Wang, a senior lecture, born in 1980 in Tongling, AnHui, has received his master degree in AnHui Polytechnic University. His main research areas are electric automation and algorithm design and improvement. 
International Journal of Signal Processing, Image Processing and Pattern Recognition Vol. 8, No. 1 (2015) 